\title{
Impact of interval from breast conserving surgery to radiotherapy on local recurrence in older women with breast cancer: retrospective cohort analysis
}

\author{
Rinaa S Punglia, assistant professor, ${ }^{1}$ Akiko M Saito, research fellow, ${ }^{2}$ chief, ${ }^{3}$ Bridget A Neville, programmer, ${ }^{2}$ \\ Craig C Earle, associate professor, ${ }^{2}$ senior scientist, ${ }^{4}$ Jane C Weeks, professor ${ }^{2}$
}

${ }^{1}$ Department of Radiation Oncology, Dana-Farber Cancer Institute, Boston, MA 02115, USA

${ }^{2}$ Department of Medical Oncology, Dana-Farber Cancer Institute,

Boston

${ }^{3}$ Laboratory of Clinical, Epidemiological and Health Services Research, Clinical Research Center, National Hospital Organization Nagoya Medical Center, Aichi, Japan

${ }^{4}$ Institute for Clinical Evaluative Sciences, Toronto, ON, Canada Correspondence to: R S Punglia rpunglia@|roc.harvard.edu

Cite this as: $B M J$ 2010;340:с845 doi:10.1136/bmj.c845

\section{ABSTRACT}

Objectives To determine if the length of interval between breast conserving surgery and start of radiotherapy affects local recurrence and to identify factors that might be associated with delay in older women with breast cancer.

Design Retrospective cohort analysis with Cox proportional hazards models to study the association between time to radiotherapy and local recurrence, and propensity score and instrumental variable analyses to confirm findings. Logistic regression investigated factors associated with later start of radiotherapy.

Setting Linked database (Surveillance, Epidemiology, and End Results Program-Medicare) in the United States Participants 18050 women aged over 65 with stage 0-II breast cancer diagnosed in 1991-2002 who received breast conserving surgery and radiotherapy but not chemotherapy.

Main outcome measure Local recurrence.

Results Median time from surgery to start of radiotherapy was 34 days, with $29.9 \%$ ( $n=5389)$ of women starting radiotherapy after six weeks. Just over $4 \%(n=734)$ of the cohort experienced a local recurrence. After adjustment for clinical and sociodemographic factors, intervals over six weeks were associated with increased likelihood of local recurrence (hazard ratio 1.19, 95\% confidence interval 1.01 to $1.39, \mathrm{P}=0.033)$. When the interval was modelled continuously (assessing accumulation of risk by day), the effect was statistically stronger (hazard ratio 1.005 per day, 1.002 to $1.008, P=0.004$ ). Propensity score and instrumental variable analysis confirmed these findings. Instrumental variable analysis showed that intervals over six weeks were associated with a $0.96 \%$ increase in recurrence at five years $(P=0.026)$. In multivariable analysis, starting radiotherapy after six weeks was significantly associated with positive nodes, comorbidity, history of low income, Hispanic ethnicity, non-white race, later year of diagnosis, and residence outside the southern states of the US.

Conclusions There is a continuous relation between the interval from breast conserving surgery to radiotherapy and local recurrence in older women with breast cancer, suggesting that starting radiotherapy as soon as possible could minimise the risk of local recurrence. There are considerable disparities in time to starting radiotherapy after breast conserving surgery. Regions of the US known to have increased rates of breast conserving surgery had longer intervals before radiotherapy, suggesting limitations in capacity. Given the known negative impact of local recurrence on survival, mechanisms to ameliorate disparities and policies regarding waiting times for treatment might be warranted.

\section{INTRODUCTION}

The past two decades have witnessed an increase in the use of radiotherapy and in waiting times to start radio therapy. ${ }^{1-4}$ Waiting lists for radiotherapy are now common in many parts of the world. ${ }^{4-8}$ Longer times to radiotherapy are a result of rise in demand-from intensified screening, greater number of diagnoses of cancer in an ageing population, and expanding indications for and increasing technological complexity of treatment - that outstrips the supply of equipment and services.

Although four to six weeks is generally cited as a reasonable interval between surgery and radiotherapy, ${ }^{9}$ evidence regarding the effect of waiting times in patients in breast cancer is mixed. Some studies have found no association between time to radiotherapy and local recurrence after breast conserving surgery, ${ }^{10-12}$ while others have reported increased recurrence among patients with longer intervals. ${ }^{13-16}$ Because local recurrence after breast conserving surgery and radiotherapy is a relatively uncommon event, the inconsistency across studies might reflect variable power to detect a difference. Moreover, many of these studies dichotomised time to radiotherapy in their analysis but used different thresholds, leaving unanswered the question of whether there is a point before which it is safe to defer radiotherapy, or if the relation between interval and recurrence is continuous.

The need for a clear answer regarding whether interval to radiotherapy affects local recurrence is especially critical given that meta-analyses of randomised studies have now unequivocally shown a link between local recurrence and decreased survival in breast cancer. ${ }^{17}$ 
A trial randomising patients to different waiting periods after surgery would provide the most definitive evidence regarding the effect of the interval from surgery to radiotherapy on local recurrence in breast cancer but would be unethical. We therefore used the linked Surveillance, Epidemiology, and End Results Program-Medicare database, the largest US population based data source within oncology, to describe the relation between interval to radiotherapy and recurrence of breast cancer.

\section{METHODS}

\section{Data sources}

The linked database we used contains information from the Surveillance, Epidemiology, and End Results Program up to 2002 and Medicare claims up to 2005. Medicare is a social insurance programme administered by the US government that provides health insurance for people aged 65 and older, those with end stage renal disease, and some people with disabilities. Medicare operates as a single payer healthcare system and is the primary health insurer for $97 \%$ of the population aged 65 and older. Medicare claims are the only national population based data in the United States that reliably and exhaustively capture medical treatment and outcomes and have therefore been used extensively to study the quality and outcomes of health care. The Surveillance, Epidemiology, and End Results Program of the National Cancer Institute is the authoritative source of information on incidence of and survival from cancer in the US. The programme collects information on tumours and demographics for all incident cancer cases in people living in California, Connecticut, Hawaii, Iowa, Kentucky, Louisiana, New Jersey, New Mexico, Utah, rural Georgia, and metropolitan areas of Atlanta, Detroit, and Seattle. New Jersey, Kentucky, Louisiana, and parts of California were in the programme from 2000 . The programme registries, which cover $26 \%$ of the population, were assembled by the National Cancer Institute for their quality and to facilitate adequate representation of minority populations to ensure applicability. Therefore, the population covered by the programme is comparable with the general US population with regard to measures of poverty and education. Inpatient and outpatient Medicare claims and physician, laboratory, durable medical equipment, home health, and hospice billings have been linked to programme data for patients aged over $65 .{ }^{18}$ The US Institute of Medicine identified the linked database as one of the few population based data resources available for analyses of quality of cancer care, and the programme population well represents the US population. ${ }^{19}$ Sociodemographic information at both the census tract and zip code level (from the 2000 census) is also included.

\section{Cohort selection}

The cohort consisted of women with unilateral pathologically confirmed stage 0-II breast cancer (American Joint Committee on Cancer) diagnosed from 1 January
1991 to 31 December 2002 at the age of 65 or older. They were all living in a region covered by the Surveillance, Epidemiology, and End Results Program and had received breast conserving surgery and radiotherapy. We restricted the cohort to patients who had not received chemotherapy within six months of surgery as chemotherapy is generally administered before radiotherapy and might exert a direct effect on the risk of local recurrence. ${ }^{20}$

We excluded patients enrolled in Medicare for renal disease or disability as they might differ from those eligible for Medicare on the basis of age. We also removed those with previous cancers, death dates that differed by more than three months in the two data sources, diagnoses made from death certificates or autopsy, and missing dates of diagnosis. Continuous Medicare (part A and part B) cover and no enrolment in a health maintenance organisation were required from month of diagnosis through to death or the end of the study (31 December 2005).

The cohort was restricted to patients who underwent radiotherapy within six months after their last breast conserving surgery. To avoid misclassification, we excluded women who had a subsequent diagnosis of cancer, local recurrence, or mastectomy within this six month period. We also restricted the analysis to those patients who survived at least two years after radiotherapy (96.6\% of the cohort) to ensure sufficient time for diagnosis and treatment of local recurrence.

Breast conserving surgery was identified in Medicare claims with current procedural terminology codes 19120, 19125-6, 19160, and 19162 from the American Medical Association, ICD-9-CM (international classification of diseases, ninth revision, clinical modification) procedural codes 85.20-85.23, or in hospital diagnosis related group codes 259-260. In Medicare records we identified radiotherapy with current procedural terminology radiation delivery codes 77401-77418 or ICD-9-CM procedure codes 92.2092.29. Radiotherapy planning was identified with current procedural terminology codes 77260-77399.

\section{Definition of interval and local recurrence}

We used the last date of breast conserving surgery within the interval from six months before to six months after diagnosis as the date of surgery. The start date for radiotherapy was determined by the date of the first radiotherapy delivery code. We used radiotherapy delivery dates linked to ICD-9-CM procedure codes when available and dates derived from current procedural terminology codes otherwise. To enhance the accuracy of the first radiotherapy date, we restricted the cohort to patients with a treatment planning current procedural terminology date from 21 days before to seven days after first radiotherapy delivery date. The number of days between the start date and last breast conserving surgery defined the interval from surgery to radiotherapy.

A local recurrence was defined as mastectomy in Medicare claims or a second pathologically confirmed 
Table 1/Demographic characteristics of population studied $(n=18$ 050)

\begin{tabular}{|c|c|}
\hline Characteristic & No (\%) of women \\
\hline \multicolumn{2}{|c|}{ Age at diagnosis (years): } \\
\hline$\geq 85$ & $643(3.6)$ \\
\hline$\geq 80-<85$ & $2221(12.3)$ \\
\hline$\geq 75-<80$ & $4437(24.6)$ \\
\hline$\geq 70-<75$ & $5557(30.8)$ \\
\hline$<70$ & $5192(28.8)$ \\
\hline \multicolumn{2}{|l|}{ Race: } \\
\hline White & $16570(91.8)$ \\
\hline Black & $807(4.5)$ \\
\hline Other & $673(3.7)$ \\
\hline \multicolumn{2}{|l|}{ Hispanic: } \\
\hline No & $17494(96.9)$ \\
\hline Yes & $556(3.1)$ \\
\hline \multicolumn{2}{|l|}{ Region: } \\
\hline Midwest & 3527 (19.5) \\
\hline West & $8800(48.8)$ \\
\hline Northeast & $3834(21.2)$ \\
\hline South & 1889 (10.5) \\
\hline \multicolumn{2}{|l|}{ Urban: } \\
\hline No & $1086(6.0)$ \\
\hline Yes & $16964(94.0)$ \\
\hline \multicolumn{2}{|c|}{ History of low income: } \\
\hline No & $16230(89.9)$ \\
\hline Yes & $1820(10.1)$ \\
\hline \multicolumn{2}{|l|}{ Charlson score: } \\
\hline 0 & $13860(76.8)$ \\
\hline 1 & $3146(17.4)$ \\
\hline 2 & $583(3.2)$ \\
\hline$\geq 3$ & $461(2.6)$ \\
\hline \multicolumn{2}{|c|}{ Married at diagnosis: } \\
\hline No & 8809 (48.8) \\
\hline Yes & $9241(51.2)$ \\
\hline \multicolumn{2}{|c|}{ Distance to nearest radiotherapy facility (miles): } \\
\hline$\geq 25 /$ unknown & $2125(11.8)$ \\
\hline$<25$ & $15925(88.2)$ \\
\hline
\end{tabular}

diagnosis of ipsilateral breast cancer in the Surveillance, Epidemiology, and End Results Program, as in Smith et al, ${ }^{21}$ or a secondary breast cancer diagnosis (ICD-9-CM 198.81) more than six months from the last breast conserving surgery.

\section{Patients' characteristics}

Explanatory variables used in this study included year of diagnosis, characteristics of tumour, other clinical characteristics (age at diagnosis, comorbidities), treatments received (nodal examinations, hormonal therapy), sociodemographic factors, and distance to nearest radiation treatment facility.

We included year of diagnosis (1991-2002) as a categorical and continuous variable. Age at diagnosis was categorised as $<70,70-<75,75-<80,80-<85$, and $\geq 85$ and was also studied as a continuous variable. Variables for tumour characteristics were categorised according to clinical cut offs defined a priori.
Comorbidities were identified with diagnostic billing codes for specific health conditions during the year before diagnosis with the Deyo implementation ${ }^{22}$ of the Charlson score ${ }^{23}$ and applied to inpatient and outpatient claims. ${ }^{24}$ The Charlson score was then categorised as $0,1,2$, or $\geq 3$. Patients without any comorbidity claims during the 13 months before diagnosis were classified as having no comorbidity. We performed a sensitivity analysis excluding these patients to ensure that results did not change. Nodal examination and use of hormonal therapy were identified from the Surveillance, Epidemiology, and End Results Program.

To measure socioeconomic status we prioritised census tract median income and per capita income over zip code to develop fifths. As an individual measure, we classified a patient as having a "personal history of low income" if she was part of the state buy-in insurance programme during 1986-2005. We categorised the cancer registries into regions of the US: west (California, Hawaii, New Mexico, Seattle, Utah); Midwest (Detroit, Iowa), northeast (Connecticut and New Jersey), and south (Atlanta, rural Georgia, Kentucky, Louisiana). Race was classified as white, black, or other, and ethnicity as Hispanic or non-Hispanic. Distance to a radiotherapy facility was determined with an algorithm that calculates the distance between the zip code of the patient's residence and that of the closest radiotherapy facility included in the 2000 American Hospital Association's annual survey of hospitals. ${ }^{25}$

\section{Main statistical analyses}

Statistical analyses were conducted with SAS for Windows (version 9.1.3; SAS Institute, Cary, NC). We studied the influence of interval to radiotherapy on time to local recurrence of breast cancer using a Cox proportional hazards model. Patients were censored at the first evidence of distant disease, death, or end of the study (31 December 2005). The final multivariable Cox model included significant variables, as well as factors identified a priori as potential predictors: treatment year, age at diagnosis, and pathology (preinvasive versus invasive). We studied two definitions of time to radiotherapy - interval dichotomised at six weeks and days to radiotherapy - in separate multivariable models. We chose six weeks as the threshold interval as it represents a cited benchmark for the initiation of radio therapy. ${ }^{926}$ We also examined interval dichotomised at different thresholds in two week increments.

In sensitivity analyses, we examined the effect of excluding patients with preinvasive carcinoma (stage 0 ) or ductal carcinoma in situ. To gain insight into potential factors that lengthen the interval to radiotherapy, we performed multivariable logistic regression using a forwards and backwards elimination algorithm to determine the final multivariable model. We did not include receipt of hormone therapy, as identified by the Surveillance, Epidemiology, and End Results Program, as an explanatory variable in this analysis as reasons for omission could be related 
Table 2|Characteristics of cancer and treatment in population studied $(n=18050)$

\begin{tabular}{|c|c|}
\hline Characteristic & No (\%) of women \\
\hline \multicolumn{2}{|l|}{ Stage: } \\
\hline $0 / \mathrm{DCIS}$ & $3058(16.9)$ \\
\hline 1 & $12171(67.4)$ \\
\hline II & $2821(15.6)$ \\
\hline \multicolumn{2}{|l|}{ Tumour size $(\mathrm{cm})$ : } \\
\hline $0-2$ & $14971(82.9)$ \\
\hline$>2-5$ & 1967 (10.9) \\
\hline$>5$ & $115(0.6)$ \\
\hline Not stated & $997(5.5)$ \\
\hline \multicolumn{2}{|l|}{ No of affected nodes: } \\
\hline 0 & $11111(61.6)$ \\
\hline 1-3/affected, but number not known & $1130(6.3)$ \\
\hline$\geq 4$ & $101(0.6)$ \\
\hline Unknown & $54(0.3)$ \\
\hline Not examined & $5654(31.3)$ \\
\hline \multicolumn{2}{|l|}{ Oestrogen receptor status: } \\
\hline Positive & $12077(66.9)$ \\
\hline Borderline & $28(0.2)$ \\
\hline Negative & $1239(6.9)$ \\
\hline Not done & $4706(26.1)$ \\
\hline \multicolumn{2}{|l|}{ Progesterone receptor status: } \\
\hline Positive & $10081(55.9)$ \\
\hline Borderline & $100(0.6)$ \\
\hline Negative & $2928(16.2)$ \\
\hline Not done & $4941(27.4)$ \\
\hline \multicolumn{2}{|l|}{ Tumour grade: } \\
\hline Well differentiated & $4515(25.0)$ \\
\hline Moderately differentiated & $7090(39.3)$ \\
\hline Poorly differentiated & $3043(16.9)$ \\
\hline Unknown & $3402(18.9)$ \\
\hline \multicolumn{2}{|l|}{ Histology: } \\
\hline Ductal & $12165(67.4)$ \\
\hline Lobular & $1329(7.4)$ \\
\hline Mixed ductal/lobular & $1127(6.2)$ \\
\hline Tubular/medullary/mucinous/papillary & $1546(8.6)$ \\
\hline Unspecified adenocarcinoma & $1232(6.8)$ \\
\hline Other histology & $651(3.6)$ \\
\hline \multicolumn{2}{|l|}{ Laterality: } \\
\hline Left & $9094(50.4)$ \\
\hline Right & $8956(49.6)$ \\
\hline \multicolumn{2}{|l|}{ Year of diagnosis: } \\
\hline 1991 & $277(1.5)$ \\
\hline 1992 & $551(3.1)$ \\
\hline 1993 & $675(3.7)$ \\
\hline 1994 & $803(4.5)$ \\
\hline 1995 & $1019(5.7)$ \\
\hline 1996 & $1151(6.4)$ \\
\hline 1997 & $1332(7.4)$ \\
\hline 1998 & 1419 (7.9) \\
\hline 1999 & $1551(8.6)$ \\
\hline 2000 & $2913(16.1)$ \\
\hline 2001 & $3136(17.4)$ \\
\hline 2002 & $3223(17.9)$ \\
\hline \multicolumn{2}{|l|}{ Hormone use: } \\
\hline No & $10935(60.6)$ \\
\hline Yes & $7101(39.4)$ \\
\hline
\end{tabular}

to factors contributing to a longer interval. We also carried out a secondary analysis studying the interaction of treatment year with regions of the US.

\section{Confirmatory analyses}

We performed a propensity score analysis to assess the consistency of the association between interval to radiotherapy and local recurrence and to control for possible differences in the distribution of confounders between the group of women who experienced increased time to radiation and those who did not. All available variables were used to create a propensity score to predict the likelihood of having an interval greater than six weeks. We studied the influence of time to radiotherapy with models that controlled for this score and performed the analyses separately within similar propensity strata.

To further assess the validity of our findings and adjust for unmeasured confounders, we also performed an instrumental variable analysis, an econometric method used to remove the effects of hidden bias in observational studies. ${ }^{27}$ An instrumental variable must fulfil two criteria: correlation with treatment (time to radiotherapy in our analysis) and lack of independent affect on outcome (recurrence in our analysis). Instead of directly comparing patients who experience delay with those who do not, which might result in bias, the instrumental variable then acts to "randomise" patients into groups that differ in their likelihood of experiencing treatment delay. Instrumental variable models provide adjusted effect estimates at one time point and on an absolute rather than a relative scale.

We selected distance to radiotherapy facility as our instrumental variable and restricted the analysis to patients with known travel distance. For the dichotomous outcome required by this analysis, we chose local recurrence by five years and restricted the analysis to patients with five years of follow-up ( $\mathrm{n}=9053)$. We separated patients into two groups, based on whether they lived within or further than 4.79 miles (about 8 $\mathrm{km}$ ) (median travel distance) of the nearest radiation facility. Patients living closer were less likely to have an interval to radiotherapy of over six weeks $(25.2 \% v$ $27.5 \%, \mathrm{P}=0.011)$. For this analysis, we used a non-parametric two stage least squares model to predict longer interval to radiotherapy. We then used local recurrence by five years as the outcome of interest and controlled for all factors significant in the proportional hazards model for local recurrence. In this technique, outcomes are reported for "marginal" patients - those expected to experience longer time to radiotherapy if they lived further from a radiation facility but not if they lived close to the treatment facility.

\section{RESULTS}

Among 18050 women with stage 0-II breast cancer (16.9\% stage $0,67.4 \%$ stage I, $15.6 \%$ stage II) who underwent breast conserving surgery, the median time from last breast surgery to start of radiotherapy was 34 days (range 1-181) and the median follow-up 
Table 3 |Factors significantly associated with local recurrence in multivariable regression analysis in addition to age and preinvasive or in situ (stage 0) carcinoma

\begin{tabular}{|c|c|c|}
\hline Variable & $\begin{array}{l}\text { Hazard ratio* } \\
\qquad(95 \% \mathrm{Cl})\end{array}$ & $P$ value \\
\hline Interval >6 weeks: & 1.19 (1.01 to 1.39$)$ & 0.033 \\
\hline Age (per each additional year of age) & 0.99 (0.98 to 1.01$)$ & 0.198 \\
\hline \multicolumn{3}{|l|}{ Hispanic: } \\
\hline No & 1.0 (reference) & \multirow{2}{*}{0.006} \\
\hline Yes & 1.61 (1.14 to 2.27$)$ & \\
\hline \multicolumn{3}{|l|}{ Tumour grade: } \\
\hline Low & 1.0 (reference) & \multirow{4}{*}{$<0.001$} \\
\hline Intermediate & 1.39 (1.12 to 1.73$)$ & \\
\hline High & 1.75 (1.37 to 2.23$)$ & \\
\hline Unknown & 1.52 (1.19 to 1.95$)$ & \\
\hline \multicolumn{3}{|l|}{ Progesterone receptor status: } \\
\hline Positive & 1.0 (reference) & \multirow{4}{*}{0.014} \\
\hline Negative & 1.37 (1.13 to 1.66$)$ & \\
\hline Borderline & 1.67 (0.79 to 3.53$)$ & \\
\hline Unknown & $1.04(0.85$ to 1.27$)$ & \\
\hline \multicolumn{3}{|l|}{ Histology: } \\
\hline Ductal & 1.0 (reference) & \multirow{6}{*}{0.010} \\
\hline Lobular & $1.00(0.75$ to 1.35$)$ & \\
\hline Mixed ductal/lobular & 1.07 (0.78 to 1.48$)$ & \\
\hline $\begin{array}{l}\text { Tubular/medullary/mucinous/ } \\
\text { papillary }\end{array}$ & $1.26(0.97$ to 1.63$)$ & \\
\hline Unspecified adenocarcinoma & 1.61 (1.24 to 2.08$)$ & \\
\hline Other histology & $0.83(0.48$ to 1.46$)$ & \\
\hline \multicolumn{3}{|l|}{ Preinvasive or in situ (stage 0) carcinoma: } \\
\hline No & 1.0 (reference) & \multirow{2}{*}{0.419} \\
\hline Yes & 1.11 (0.86 to 1.42$)$ & \\
\hline Year (per each later year of diagnosis) & 0.95 (0.92 to 0.97$)$ & $<0.001$ \\
\hline \multicolumn{3}{|l|}{ Laterality: } \\
\hline Right & 1.0 (reference) & \multirow{2}{*}{0.016} \\
\hline Left & $1.20(1.03$ to 1.38$)$ & \\
\hline
\end{tabular}

time was 5.38 years (2.0-14.9). Tables 1 and 2 describe patients' characteristics.

During follow-up 734 (4\%) women experienced local recurrences after radiotherapy. An adjusted Cox model showed that an interval to radiotherapy of over six weeks was significantly associated with an increased hazard for local recurrence (hazard ratio $1.19,95 \%$ confidence interval 1.01 to $1.39, \mathrm{P}=0.033$ ). Other factors independently associated with local recurrence included Hispanic ethnicity, tumour histology, left sided laterality, lack of hormone receptor expression, increased tumour grade, and earlier year of diagnosis (table 3). When we adjusted for the same variables, the interval defined as days to start of radiotherapy was more strongly correlated with local recurrence $(\mathrm{P}=0.004)$ (table 4$)$. Adjusted hazard ratios with interval dichotomised at different thresholds supported a continuous relation between interval and local recurrence (fig 1). Results did not change significantly when we excluded patients with stage 0 cancer (table 4) or those with missing comorbidity calculation (hazard ratio $1.18,1.01$ to $1.39, \mathrm{P}=0.041$ ).
Confirmatory analyses suggested that the results were also unchanged when we added a propensity score predicting likelihood of receiving radiotherapy after six weeks to the model $(1.19,1.01$ to 1.39 , $\mathrm{P}=0.035)$, or when we studied the effect of interval to radiotherapy within similar propensity strata. In addition, adjusted instrumental variable analysis showed that starting radiotherapy after six weeks was associated with a $0.96 \%$ absolute increase in local recurrence $(\mathrm{P}=0.026)$.

Over a quarter $(26.3 \%)$ of the overall cohort waited more than six weeks from last breast surgery to start of radiotherapy. In multivariable analysis (table 5), greater comorbidity, being non-white, Hispanic ethnicity, residence outside of the southern states, history of low income, having affected nodes, and more recent year of diagnosis were independently associated with starting radiotherapy after six weeks. Secondary analysis showed significant interaction between region of the country and treatment year $(\mathrm{P}<0.001$; fig 2$)$.

\section{DISCUSSION}

\section{Summary of findings}

In older women with breast cancer who do not receive chemotherapy the interval from breast conserving surgery to radiotherapy affects the risk of local recurrence. Women who start radiotherapy more than six weeks after surgery are more likely to have local recurrence. The association is stronger when the interval is measured as days to radiotherapy, rather than dichotomised at six weeks. This finding suggests that there is no particular threshold, but instead a continuous relation between time to radiotherapy and local recurrence. Because the absolute risk of local recurrence is low, however, differences in the rates of recurrence are small. Our instrumental variable analysis showed that intervals over six weeks are associated with a $0.96 \%$ increase in recurrence at five years.

\section{Comparison with other studies}

Previous studies that have examined interval to radiotherapy as a dichotomous variable have also provided

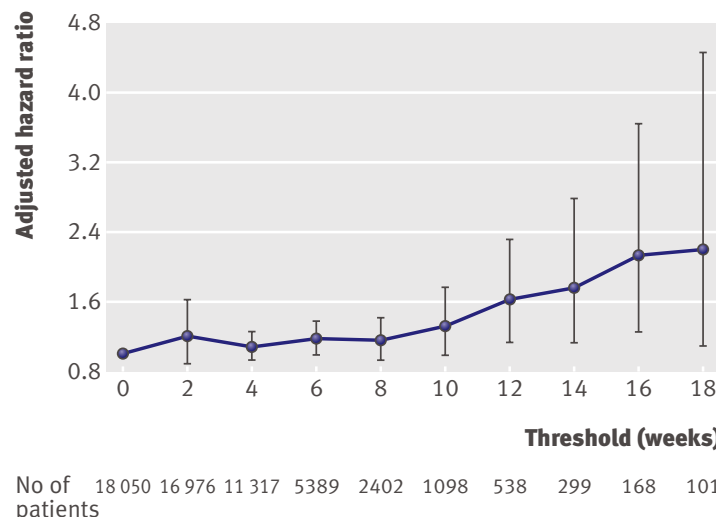
patients

Fig 1| Adjusted hazard ratios for local recurrence with varying dichotomous thresholds. Numbers below graph reflect patients who received radiotherapy after threshold 
Table 4 Adjusted hazard ratios for local recurrence in multivariable analyses

\begin{tabular}{|c|c|c|c|c|}
\hline \multirow[b]{2}{*}{ Definition of interval } & \multicolumn{2}{|l|}{ Stage 0 -II } & \multicolumn{2}{|l|}{ Stage I-II } \\
\hline & $\mathrm{HR}(95 \% \mathrm{Cl})$ & $\begin{array}{c}P \\
\text { value }\end{array}$ & $\mathrm{HR}(95 \% \mathrm{Cl})$ & $P$ value \\
\hline$>6$ weeks & 1.19 (1.01 to 1.39$)$ & 0.033 & 1.14 (0.96 to 1.36$)$ & 0.139 \\
\hline Days to start of radiotherapy & $1.005(1.002$ to 1.008$)$ & 0.004 & 1.005 (1.001 to 1.009$)$ & 0.016 \\
\hline
\end{tabular}

some indirect evidence that the relation might actually be continuous. Across these studies, the effect of time to radiotherapy on local recurrence increased the longer the interval examined, consistent with our observation of increased hazard with longer thresholds (fig 1). For example, the hazard ratio in our analysis with a threshold of six weeks was 1.19 (1.01 to 1.39). A meta-analysis with a longer threshold of 9-16 weeks after surgery found increased odds of 1.62 (1.21 to 2.16) for recurrence relative to patients treated within eight weeks. ${ }^{14} \mathrm{~A}$ population based study in Quebec that examined the longer threshold of 12 weeks after surgery was associated with a higher odds ratio for local failure of 1.75 (1.00 to 3.08). ${ }^{16}$ And a population based study from British Columbia found a hazard ratio of 2.00 (0.79 to 5.08) for local recurrence in women who received radiotherapy after 20 weeks. Because of our larger sample size, our analysis had greater power to detect small differences in recurrence than previous studies, thus allowing us to identify a significant difference associated with shorter delays. ${ }^{13-16}$ Our study also provides further insight into the nature of the relation between interval and recurrence - namely, that the effect is continuous without a single threshold time.

\section{Implications for survival}

Because of the long natural course of early breast cancer, our mean follow-up of 5.4 years was not sufficient to allow us to examine the effect of time to radiotherapy on survival. Differences in mortality attributable to local recurrence in breast cancer cannot be detected until 10-15 years after treatment. ${ }^{17}$ Randomised studies, however, support a robust association between local recurrence and overall survival in breast cancer and suggest that for every four local recurrences prevented through improved local treatment, one death from breast cancer is avoided. ${ }^{17}$ Therefore, our findings with respect to the effect of longer interval to radiotherapy on increased local recurrence could lead to compromised survival.

\section{Strengths and limitations}

Because of its size, the linked dataset gave us greater power to detect an association between time to radiotherapy and local recurrence, but it also limited our analysis to patients aged over 65 . It seems reasonable to hypothesise that our findings might be generalisable to younger women. Previous studies that included younger women yielded consistent results. ${ }^{13}{ }^{16}$ Furthermore, a meta-analysis of radiotherapy after breast conserving surgery showed similar proportional reductions across age groups. ${ }^{17}$ In fact, because younger age has been shown to be an independent risk factor for local recurrence after breast conserving surgery, ${ }^{28}$ the association between interval to radiotherapy and local recurrence could be even more pronounced in younger patients.

Medicare claims are submitted for billing not research purposes, and the determination of interval to radiotherapy might not be precise. We attempted to increase the accuracy of the definition of interval to radiotherapy by restricting the analysis to those with a treatment planning billing date from three weeks before to seven days after initiation of radiotherapy, but in doing so we might have excluded radiation departments with delays in the treatment planning process. We also had no information about the status of the surgical margin and dose of radiation, both of which might modulate the effect of interval on local recurrence. There is some evidence that women with affected margins might receive higher doses of radiation and receive radiotherapy closer to time of surgery. ${ }^{11}$ If this were true in our cohort, however, our results would be biased towards the null and our detected association between interval and local recurrence would be underestimated. Moreover, we controlled for year of diagnosis in our analysis to mitigate the effect of any secular trends in evaluation and status of the surgical margin.

In addition, because local recurrence after radiotherapy is a relatively rare event, we included women with stage 0 and node positive disease to increase the power of our analysis. Our results were essentially unchanged when we restricted the analysis to women with invasive disease. Our main outcome measure, local recurrence, was largely identified by a mastectomy procedure requiring women to be well enough to undergo surgery for local recurrence to be captured and might therefore not be completely sensitive for capture of local recurrence. We attempted to help to ensure capture of our outcome and increase the likelihood of receiving adequate initial treatment by limiting the analyses to those women who survived two years from radiotherapy, and recurrence rates in our analysis are comparable with those reported for true recurrences with radiotherapy after breast conserving

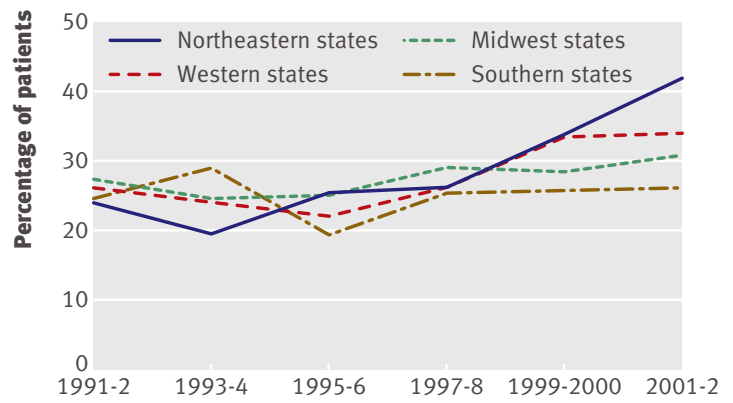

Year

Fig 2 Regional variation in percentage of patients with interval longer than six weeks over period studied 
Table 5|Factors significantly associated with interval to radiotherapy of over six weeks in multivariable logistic regression analysis

\begin{tabular}{|c|c|c|}
\hline Variable & $\begin{array}{l}\text { Odds ratio } \\
(95 \% \mathrm{Cl})\end{array}$ & $P$ value \\
\hline \multicolumn{3}{|l|}{ Charlson score: } \\
\hline 0 & 1.0 (reference) & \multirow{4}{*}{0.030} \\
\hline 1 & 1.11 (1.02 to 1.21$)$ & \\
\hline 2 & $1.12(0.93$ to 1.33$)$ & \\
\hline$\geq 3$ & 0.89 (0.72 to 1.09$)$ & \\
\hline \multicolumn{3}{|l|}{ Race: } \\
\hline White & 1.0 (reference) & \multirow{3}{*}{$<0.001$} \\
\hline Black & 1.45 (1.24 to 1.68$)$ & \\
\hline Other & 0.94 (0.79 to 1.12$)$ & \\
\hline \multicolumn{3}{|l|}{ Hispanic: } \\
\hline No & 1.0 (reference) & \multirow{2}{*}{0.006} \\
\hline Yes & $1.29(1.08$ to 1.54$)$ & \\
\hline \multicolumn{3}{|l|}{ State buy-in: } \\
\hline No & 1.0 (reference) & $<0.001$ \\
\hline Yes & 1.27 (1.15 to 1.42$)$ & \\
\hline \multicolumn{3}{|l|}{ Region: } \\
\hline South & 1.0 (reference) & \multirow{4}{*}{$<0.001$} \\
\hline Northeast & 1.66 (1.46 to 1.88$)$ & \\
\hline Midwest & 1.31 (1.15 to 1.49$)$ & \\
\hline West & 1.35 (1.20 to 1.52$)$ & \\
\hline \multicolumn{3}{|l|}{ Positive nodes: } \\
\hline 0 & 1.0 (reference) & \multirow{5}{*}{$<0.001$} \\
\hline $1-3$ or unknown & 1.21 (1.06 to 1.37$)$ & \\
\hline$\geq 4$ & $1.23(0.81$ to 1.87$)$ & \\
\hline Not examined & $0.76(0.70$ to 0.81$)$ & \\
\hline Unknown & 0.91 (0.79 to 1.66$)$ & \\
\hline Per each additional year after 1991 & 1.07 (1.05 to 1.08$)$ & $<0.001$ \\
\hline
\end{tabular}

surgery. ${ }^{29}$ Additionally, although the linked database intentionally oversamples minority populations, our study cohort had fewer minorities than the overall US population, consistent with the lower average age and decreased likelihood of receipt of breast conserving surgery and radiotherapy among non-white US populations. $^{30-32}$

We did not study the question of timing of radiotherapy relative to chemotherapy. A randomised trial varying the sequence of chemotherapy and radiotherapy, however, did not find any difference in local recurrence between the groups ${ }^{33}$ suggesting that the effect of time from surgery to radiation might be comparable with the time from completion of chemotherapy to the start of radiotherapy. Additionally, many of the women in our dataset did not receive axillary nodal surgery, evaluation of hormonal status, or histological grading consistent with other analyses of practice patterns during the era studied. ${ }^{34}$ These omissions could also indicate that some women did not receive optimal radiotherapy. We controlled for year in our analysis to mitigate the effect of bias caused by secular trends in improved practice patterns. Beyond previous participation in state based insurance plans targeted at low income patients, we did not have individual measures of socioeconomic status, although we did test the significance of ecological measures of socioeconomic status at the census tract level. Finally, although we attempted to discern any effect of bias on our results by using three different modelling techniques, we cannot exclude the possibility that there might be additional factors associated with both time to start of radiotherapy and local recurrence, which our dataset did not enable us to study and which might confound our results.

\section{Sources of delay}

The best intervention to reduce delay depends on the factors responsible for that delay. Difficulties at the patient level might be addressed through navigation programmes. In contrast, inefficient processes in pathology review or referral might best be addressed through institutional quality improvement efforts in multidisciplinary coordination. Finally, limitation in radiotherapy capacity would probably require investments to increase staff and facilities, or possibly techniques to increase existing capacity such as hypofractionation or brachytherapy.

Our analysis of factors associated with increased time to radiotherapy might provide insight into one source of delay. The use of breast conserving surgery increased during the period studied, ${ }^{3135}$ and we found that more recent year of diagnosis was a significant predictor of increased interval to radiotherapy, but the effect varied by region of the US. In the northeast, the proportion of women whose radiation started more than six weeks after surgery almost doubled, from $23.8 \%$ during $1991-2$ to $42.0 \%$ during $2001-2$. In contrast, in the southern states of the US, where the rates of breast conservation were the lowest, ${ }^{30}$ there was little or no increase in the proportion of women receiving radiation after six weeks over the same period. Longer times to radiotherapy during recent years and in regions of the US known to have increased use of breast conserving surgery suggest limitations in capacity of radiation delivery. Indeed, our instrumental variable analysis showed an association between interval to radiotherapy and longer distances to treatment facility, providing further indirect evidence that lack of availability of radiotherapy is contributing to delays.

\section{Conclusions and policy implications}

The implication of a continuous relation between start of radiotherapy and local recurrence is that there is no "safe" threshold in terms of waiting time and that radiotherapy should therefore be started as soon as possible. The cost of increasing capacity to ensure uniformly short waiting times could be substantial. These costs would need to be weighed against the small absolute benefit in local recurrence that might be the result of the investment. But given the known negative impact of local recurrence on overall survival and the large numbers of women treated with radiotherapy for breast cancer, it seems appropriate to consider whether this is a price we should be prepared to pay. 


\section{WHAT IS ALREADY KNOWN ON THIS TOPIC}

Previous studies regarding the effect of interval to radiotherapy in women treated for breast cancer have had mixed findings

\section{WHAT THIS STUDY ADDS}

Using a large population based database, we found an increase in the risk of local recurrence with longer interval to radiotherapy

The relation between interval to radiation and local recurrence seems to be continuous

We thank E Francis Cook, Harvard School of Public Health, for his generous methodological support. The interpretation and reporting of these data are the sole responsibility of the authors. The authors acknowledge the efforts of the Applied Research Program, National Cancer Institute; the Office of Research, Development and Information, CMS; Information Management Services; and the Surveillance,

Epidemiology, and End Results Program tumor registries in the creation of the linked database.

Contributors: RSP, CCE, and JCW were involved in the conception and design of the study. BAN created the dataset. RSP and JCW performed the analysis and data interpretation. AMS directed the confirmatory statistica analyses. RSP and JCW drafted the article. All authors were involved in the critical revision of the manuscript and gave approval of the version submitted. RSP is guarantor.

Funding: This study was supported in part by a grant from the National Institutes of Health (1K07 CA118629 to RSP) and from the American Society of Clinical Oncology (Career Development Award to RSP). It was first presented in part at the annual meeting of the American Society of Clinical Oncology, June 2008.

Competing interests: None declared.

Ethical approval: This study was reviewed and approved by the institutional review board of Dana-Farber Cancer Institute/Partners Cancer Care (DFCl 06-391).

Data sharing: No additional data available.

1 Jack RH, Davies EA, Robinson D, Sainsbury R, Moller H. Radiotherapy waiting times for women with breast cancer: a population-based cohort study. BMC Cancer 2007;7:71.

2 Mikeljevic JS, Haward R, Johnston C, Crellin A, Dodwell D, Jones A, et al. Trends in postoperative radiotherapy delay and the effect on survival in breast cancer patients treated with conservation surgery. Br J Cancer 2004;90:1343-8.

3 Johnston GM, MacGarvie VL, Elliott D, Dewar RA, Maclntyre MM, Nolan MC. Radiotherapy wait times for patients with a diagnosis of invasive cancer, 1992-2000. Clin Invest Med 2004;27:142-56.

4 Mackillop WJ, Fu H, Quirt CF, Dixon P, Brundage M, Zhou Y. Waiting for radiotherapy in Ontario. Int J Radiat Oncol Biol Phys 1994;30:221-8

5 Dodwell D, Crellin A. Waiting for radiotherapy. BMJ 2006;332:107-9.

6 Schäfer C, Nelson K, Herbst M. Waiting for radiotherapy. Strahlentherapie und Onkologie 2005;181:9-19.

7 Lim KSH, Vinod SK, Bull C, O’Brien P, Kenny L. Prioritization of radiotherapy in Australia and New Zealand. Australas Radiol 2005;49:485-8.

8 Mackillop WJ. Killing time: the consequences of delays in radiotherapy. Radiother Oncol 2007;84:1-4.

9 Buchholz TA. Radiation therapy for early-stage breast cancer after breast-conserving surgery. N Engl J Med 2009;360:63-70.

10 Cefaro GA, Genovesi D, Marchese R, Di Tommaso M, Di Febo F, Ballone $\mathrm{E}$, et al. The effect of delaying adjuvant radiation treatment after conservative surgery for early breast cancer. Breast J 2007;13:575-80.

11 Froud PJ, Mates D, Jackson JS, Phillips N, Andersen S, Jackson SM, et al. Effect of time interval between breast-conserving surgery and radiation therapy on ipsilateral breast recurrence. Int / Radiat Oncol Biol Phys 2000;46:363-72.

12 Livi L, Borghesi S, Saieva C, Meattini I, Rampini A, Petrucci A, et al. Radiotherapy timing in 4,820 patients with breast cancer: University of Florence experience. Int J Radiat Oncol Biol Phys 2009;73:365-9.
13 Olivotto IA, Lesperance ML, Truong PT, Nichol A, Berrang T, Tyldesley S, et al. Intervals longer than 20 weeks from breastconserving surgery to radiation therapy are associated with inferior outcome for women with early-stage breast cancer who are not receiving chemotherapy. J Clin Oncol 2009;27:16-23.

14 Huang J, Barbera L, Brouwers M, Browman G, Mackillop WJ. Does delay in starting treatment affect the outcomes of radiotherapy? A systematic review. J Clin Oncol 2003;21:555-63.

15 Chen Z, King W, Pearcey R, Kerba M, Mackillop WJ. The relationship between waiting time for radiotherapy and clinical outcomes: a systematic review of the literature. Radiother Oncol 2008;87:3-16.

16 Hebert-Croteau N, Freeman CR, Latreille J, Rivard M, Brisson J. A population-based study of the impact of delaying radiotherapy after conservative surgery for breast cancer. Breast Cancer Res Treat 2004;88:187-96.

17 Clarke M, Collins R, Darby S, Davies C, Elphinstone P, Evans E, et al. Effects of radiotherapy and of differences in the extent of surgery fo early breast cancer on local recurrence and 15-year survival: an overview of the randomised trials. Lancet 2005;366:2087-106.

18 Potosky AL, Riley GF, Lubitz JD, Mentnech RM, Kessler LG. Potential for cancer related health services research using a linked Medicaretumor registry database. Med Care 1993;31:732-48.

19 Warren JL, Klabunde CN, Schrag D, Bach PB, Riley GF. Overview of the SEER-Medicare data: content, research applications, and generalizability to the United States elderly population. Med Care 2002;40(suppl 8):IV-3-18S

20 Early Breast Cancer Trialists' Collaborative Group (EBCTCG). Effects of chemotherapy and hormonal therapy for early breast cancer on recurrence and 15-year survival: an overview of the randomised trials. Lancet 2005;365:1687-717.

21 Smith BD, Gross CP, Smith GL, Galusha DH, Bekelman JE, Haffty BG. Effectiveness of radiation therapy for older women with early breast cancer. J Natl Cancer Inst 2006;98:681-90.

22 Deyo RA, Cherkin DC, Ciol MA. Adapting a clinical comorbidity index for use with ICD-9-CM administrative databases. J Clin Epidemiol 1992;45:613-9.

23 Charlson ME, Pompei P, Ales KL, MacKenzie CR. A new method of classifying prognostic comorbidity in longitudinal studies: development and validation. J Chronic Dis 1987;40:373-83.

24 Klabunde CN, Potosky AL, Legler JM, Warren JL. Development of a comorbidity index using physician claims data. J Clin Epidemiol 2000;53:1258-67.

25 American Hospital Association (AHA). Hospital statistics. AHA, 2000.

26 Whelan TJ. Use of conventional radiation therapy as part of breastconserving treatment. J Clin Oncol 2005;23:1718-25.

27 Newhouse JP, McClellan M. Econometrics in outcomes research: the use of instrumental variables. Annu Rev Public Health 1998;19:17-34

28 Fisher ER, Anderson S, Tan-Chiu E, Fisher B, Eaton L, Wolmark N. Fifteen-year prognostic discriminants for invasive breast carcinoma: National Surgical Adjuvant Breast and Bowel Project Protocol-06. Cancer 2001;91(suppl 8):1679-87S.

29 Gage I, Recht A, Gelman R, Nixon AJ, Silver B, Bornstein BA, et al. Long-term outcome following breast-conserving surgery and radiation therapy. Int J Radiat Oncol Biol Phys 1995;33:245-51.

30 Morrow M, White J, Moughan J, Owen J, Pajack T, Sylvester J, et al. Factors predicting the use of breast-conserving therapy in stage I and Il breast carcinoma. J Clin Oncol 2001;19:2254-62.

31 Lazovich D, Solomon CC, Thomas DB, Moe RE, White E. Breast conservation therapy in the United States following the 1990 National Institutes of Health Consensus Development Conference on the treatment of patients with early stage invasive breast carcinoma. Cancer 1999;86:628-37.

32 Ballard-Barbash R, Potosky AL, Harlan LC, Nayfield SG, Kessler LG. Factors associated with surgical and radiation therapy for early stage breast cancer in older women. / Natl Cancer Inst 1996;88:716-26.

33 Bellon JR, Come SE, Gelman RS, Henderson IC, Shulman LN, Silver BJ, et al. Sequencing of chemotherapy and radiation therapy in earlystage breast cancer: updated results of a prospective randomized trial. J Clin Oncol 2005;23:1934-40.

34 Hillner BE, Penberthy L, Desch CE, McDonald MK, Smith TJ, Retchin SM. Variation in staging and treatment of local and regiona breast cancer in the elderly. Breast Cancer Res Treat 1996;40:75-86.

35 Gilligan MA, Kneusel RT, Hoffmann RG, Greer AL, Nattinger AB. Persistent differences in sociodemographic determinants of breast conserving treatment despite overall increased adoption. Med Care 2002;40:181-9.

Accepted: 21 December 2009 